\title{
Mask Compliance Training for Individuals With Intellectual and Developmental Disabilities
}

\author{
Michelle A. Frank-Crawford ${ }^{1,2} \cdot$ Morgan M. Hallgren ${ }^{1} \cdot$ Anlara McKenzie $^{1} \cdot$ Meagan K. Gregory ${ }^{1,2}$ • \\ Margaret E. Wright ${ }^{1} \cdot$ Lee E. Wachtel ${ }^{1,3}$
}

Accepted: 24 March 2021 / Published online: 15 June 2021

(C) Association for Behavior Analysis International 2021

\begin{abstract}
Since the arrival of the novel coronavirus, recommendations for public masking have emerged to decrease infection rates. For a variety of reasons, tolerating wearing a mask is challenging for many individuals with intellectual and developmental disabilities (IDDs). Therefore, we evaluated behavioral strategies to promote compliance with wearing a mask with six hospitalized individuals diagnosed with IDDs. One participant was compliant with wearing the mask for extended durations during baseline while engaging in various activities (e.g., academics, leisure). For the other five individuals, engagement in activities alone was ineffective. Blocking mask removal, reinforcement for mask wearing, and noncontingent access to preferred activities or competing stimuli were then evaluated using a changing-criterion design in which the duration participants were required to tolerate the mask gradually increased. Increases in compliance with mask wearing were achieved with all participants; however, the terminal duration was attained for only four of the five individuals.
\end{abstract}

Keywords Changing-criterion design $\cdot$ Compliance training $\cdot$ Face masks $\cdot$ COVID-19

Infectious diseases are those which are spread directly or indirectly from one person to another. Direct transmission requires that the potential host be in the presence of the infectious agent and physically contact the disease. Alternatively, indirect transmission can be achieved by a vehicle to which the disease is attached (e.g., food), vectors (e.g., mosquitos) that carry the disease within them, or respiratory droplets when the disease is airborne (Centers for Disease Control and Prevention [CDC], 2020). Those that transmit via respiratory droplets are shared when an infected person coughs, sneezes, talks, or otherwise expels aerosolized particles (Ather et al., 2020). These diseases can be particularly infectious because the droplets can remain suspended in the air

Meagan K. Gregory

Gregory@kennedykrieger.org

1 Department of Behavioral Psychology, Neurobehavioral Unit, Kennedy Krieger Institute, 707 N. Broadway, Baltimore, MD 21205 , USA

2 Department of Psychiatry and Behavioral Science, School of Medicine, Johns Hopkins University, Baltimore, MD, USA

3 Department of Child and Adolescent Psychiatry, Kennedy Krieger Institute, Baltimore, MD 21205, USA after the infected agent is no longer present, and can be blown over a great distance (CDC, 2020).

One example of an infectious disease spread via respiratory droplets is the novel coronavirus, otherwise known as SARSCoV-2 or COVID-19. It was first identified in Wuhan Province, China, at the end of 2019 and rapidly spread across countries and continents, inciting a global pandemic on March 11, 2020. At the time of writing, COVID-19 was responsible for $93,000,000$ cases and nearly 2,000,000 deaths worldwide; over 388,000 of those deaths had occurred in the United States alone (Johns Hopkins University \& Medicine, n.d.).

Health officials, such as the CDC (2020), have outlined specific practices to slow or prevent the spread of infectious diseases, such as COVID-19, including washing hands as often as possible (for at least $20 \mathrm{~s}$ each time), avoiding close contact with others both within and outside of the home, and frequently disinfecting high-touch surfaces. In addition, a face mask that simultaneously covers the mouth and nose is highly recommended for individuals over the age of 2 who are conscious, do not have a history of breathing difficulties, and can independently remove the mask. The use of masks by healthy or asymptomatic individuals in public settings has been found to potentially slow infection by reducing the transmission of 
respiratory droplets (Cheng et al., 2020). A recent metaanalysis of 172 observational studies across six continents examined interventions for halting the transmission of coronaviruses, including COVID-19, and determined that both physical distancing of greater than $1 \mathrm{~m}$ and masking reduced the risk of infection (Chu et al., 2020). Other projected models have shown that the use of face masks, even many homemade cloth models or those not of medical grade, reduces transmission and infection and may also lead to decreased peak hospitalizations and death (i.e., they help "flatten the curve" of infection; Eikenberry et al., 2020; Sunjaya \& Jenkins, 2020). Many countries have recommended or mandated public masking, with variable fidelity, particularly across the United States. Nonetheless, the practical matter remains that to gain entrance to many critical public venues, including schools, supermarkets, retail stores, and more recently entertainment venues, wearing a mask has become de rigueur.

This new reality may be irritating to those who find this extra step to be burdensome; however, for individuals with intellectual and developmental disabilities (IDDs), it may be a barrier that prevents their or their families' ability to acquire goods and services required for education, health, and wellbeing. Furthermore, infectious diseases such as COVID-19 can pose an even greater risk to those with IDDs than the general population. Recent studies have demonstrated that people with IDDs, regardless of whether they live at home or in a residential setting, have a higher risk of death from COVID-19 (Courtenay \& Perera, 2020; Shapiro, 2020; Turk et al., 2020). Separate reports compiled by the New York State Office for People with Developmental Disabilities and the Office of Developmental Programs of the Pennsylvania Department of Human Services demonstrated that individuals with IDDs are 2.5 to 4 times more likely to contract COVID-19 than their typical peers (Shapiro, 2020). Another study found that the risk of death from COVID-19 in patients aged 0-17 years was $1.6 \%$ for those with IDDs, and $<0.01 \%$ for those without such a diagnosis. In those aged 18-74 years, risk of death was $4.5 \%$ for those with IDDs, compared to $2.7 \%$ for those without IDDs (Turk et al., 2020). This increased risk for infection and death may not be surprising given that individuals with IDDs often have comorbid physical conditions (Courtenay $\&$ Perera, 2020). Furthermore, the increased risk of death is perhaps exasperated by the fact that respiratory infection was already the leading cause of death among those with IDDs, even before the COVID-19 pandemic (O'Leary et al., 2018).

It is prudent that individuals with IDDs be taught to wear masks to help decrease the possibility of infection (to themselves and others), limit the adverse effects from disruptions to routines, and increase their access to the community. This is particularly salient as the world comes to recognize that recovery from the pandemic will be a slow process taking several months to over a year. However, many such individuals may find it difficult to tolerate wearing a mask. Individuals with IDDs, in particular those with autism spectrum disorder, are reported to exhibit abnormal responses to tactile stimulation (e.g., Puts et al., 2014), such as that which may be experienced when wearing a mask. Often, individuals with IDDs are also reported to be noncompliant with medical procedures (Stuesser \& Roscoe, 2020), as well as routine health care activities (e.g., DeLeon et al., 2008). If such activities are aversive, then their presentation may come to evoke escapemaintained problem behavior. As such, increasing tolerance for health care activities, such as wearing a mask during the COVID-19 pandemic, is an important goal for individuals with IDDs.

Fortunately, previous research has shown that behavioral interventions can be very successful in building tolerance across a variety of issues, including spending time away from caregivers, consuming lesser preferred foods, wearing eyeglasses, and wearing clothing to help prevent inappropriate behavior (e.g., DeLeon et al., 2008; Flood \& Wilder, 2004; Kahng et al., 2003; Wheatley et al., 2019). Notably, many (but not all) of these referenced studies used a changing-criterion design (CCD), suggesting that it can be particularly effective when teaching individuals to tolerate potentially aversive situations (see McDougall et al., 2006, for a review). For example, Wheatley et al. (2019) used a CCD to increase the duration of wearing a leotard to help prevent access to fecal play. These studies also highlight various treatment components that may be effective in increasing compliance with health care activities. For example, using a reversal desgin, DeLeon et al. (2008) found that noncontingent reinforcement (NCR) was effective at increasing the duration of wearing glaases for one individual with IDD and the combination of NCR, response cost, and response blocking was effective for three other individuals.

Although public opinion has been diverse in terms of the necessity and efficacy of mask use, developing scientific research supports the reduction in viral transmission with mask compliance. Therefore, the purpose of the present study was to extend research on compliance with medically indicated interventions with individuals diagnosed with IDDs by building tolerance to wearing a mask during the COVID-19 pandemic. For three individuals, component analyses were also conducted to determine which components were necessary to maintain compliance with mask wearing after the terminal duration was met.

\section{Method}

\section{Participants and Setting}

Participants included six patients admitted to an inpatient unit for the assessment and treatment of severe problem behavior. Table 1 details participant characteristics, including 
Table 1 Participant Information

\begin{tabular}{|c|c|c|c|c|}
\hline Participant & $\begin{array}{l}\text { Age } \\
\text { (years) }\end{array}$ & Gender & Diagnoses & Targeted problem behavior \\
\hline Eleanor & 14 & Female & Profound ID, ASD & Aggression, disruptive behavior, SIB \\
\hline Miles & 18 & Male & $\begin{array}{l}\text { Unspecified ID, stereotypic movement disorder with SIB, disruptive behavior } \\
\text { disorder, unspecified mood and anxiety disorder, and monoallelic mutation of } \\
\text { the PACS1 gene }\end{array}$ & $\begin{array}{l}\text { Aggression, disruptive behavior, } \\
\text { inappropriate verbalizations, SIB }\end{array}$ \\
\hline Tobias & 14 & Male & Unspecified ID, disruptive behavior disorder, ASD & Aggression, disruptive behavior \\
\hline Wesley & 6 & Male & $\begin{array}{l}\text { Unspecified ID; stereotypic movement disorder with SIB; unspecified disruptive, } \\
\text { impulse-control, and conduct disorder; ASD }\end{array}$ & SIB \\
\hline Garrett & 13 & Male & $\begin{array}{l}\text { Severe ID; stereotypic movement disorder with SIB; unspecified disruptive, } \\
\text { impulse-control, and conduct disorder; ASD }\end{array}$ & Aggression, disruptive behavior, SIB \\
\hline Graham & 20 & Male & $\begin{array}{l}\text { Moderate ID, stereotypic movement disorder with SIB, unspecified disturbance of } \\
\text { conduct, ASD }\end{array}$ & Aggression, disruptive behavior, SIB \\
\hline
\end{tabular}

Note. $\mathrm{ID}=$ intellectual disability; $\mathrm{ASD}=$ autism spectrum disorder; $\mathrm{SIB}=$ self-injurious behavior.

age, gender, diagnoses, and topographies of problem behavior targeted during their inpatient admission. Participation was approved by medical personnel who worked with the patients daily; individuals were selected for inclusion based on meeting the guidelines set forth by the institute's infection control staff. Specifically, patients who were above 2 years of age, who did not have any respiratory compromise, and who could independently remove the mask were exposed to masktolerance training. Sessions were conducted by members of the participants' inpatient behavioral team. These behavioral therapists wore personal protective equipment across all sessions, consisting of surgical or cloth masks and face shields.

Sessions were conducted in an activity room measuring approximately $3.2 \mathrm{~m} \times 3.2 \mathrm{~m}$ (Miles, Tobias, Wesley, and Garrett) or across various locations of the inpatient unit (Eleanor and Graham). Rooms were equipped with chairs, tables, and other materials necessary to complete the sessions (present in various phases of the study). Other materials included cloth masks (that either tied around the participant's head or hooked over their ears) and preferred activities and foods (identified through previously conducted, separate edible and tangible preference assessments). Masks selected for inclusion were consistent with those recommended by the CDC (2020). In addition, competing stimuli were included for Eleanor, and tokens were included for Tobias and Wesley.

\section{Experimental Design, Data Collection, and Interobserver Agreement}

\section{Design}

A CCD was used to build tolerance to increasingly longer durations of wearing a cloth mask. With the exception of Tobias, the initial criterion equaled the average duration for which the mask was worn in baseline. Tobias tolerated the mask for varying durations during baseline and wore the mask for the initial goal of $15 \mathrm{~min}$ across several consecutive sessions. Therefore, the initial criterion for treatment for Tobias was $15 \mathrm{~min}$. However, the duration of wearing the mask remained variable during treatment; therefore, a second and lower initial criterion of $30 \mathrm{~s}$ was implemented. The duration for which the participants were required to wear the mask was increased across sessions. The criterion initially increased by $50 \%$ (Eleanor and Miles), doubled (Tobias and Wesley), or increased by 15 -min increments (Garrett) contingent on three consecutive sessions in which the participant met the criterion (i.e., did not successfully remove the mask prior to meeting the duration criterion). For Wesley, this was later changed to increases of 50\% (rather than doubling). Later in the evaluation, larger increases in session duration than those outlined previously were sometimes used; in addition, some phase lengths varied, with more sessions conducted at some criteria than was prescribed. The criterion continued to increase until the participant reached the terminal goal or responding remained consistently below the goal for at least double the number of sessions conducted in baseline. The terminal goal was individually determined based on the anticipated intervals of time that participants would be required to wear a mask in the community (e.g., a trip to the grocery store) or at school (e.g., the duration of a class period before receiving a break). An additional variable, age, was taken into consideration for Wesley. Specifically, nursing personnel recommended a goal of approximately $10 \mathrm{~min}$ prior to providing him a brief break from wearing the mask (e.g., time to remove the mask, take a small bite of food, and then replace the mask). The terminal duration for wearing the mask was $10 \mathrm{~min}$ (as noted) for Wesley, $15 \mathrm{~min}$ for Eleanor, $30 \mathrm{~min}$ for Miles and Tobias, and $60 \mathrm{~min}$ for Garrett and Graham.

The treatment evaluation began in the same fashion for Graham; however, he was compliant with wearing the mask for extended durations of time during baseline (e.g., upward of 
$3 \mathrm{hr}$ ), exceeding his terminal goal. Therefore, additional tolerance training was deemed unnecessary.

\section{Data Collection}

Observers collected data on laptop computers equipped with BDataPro (Bullock et al., 2017) for Eleanor, Tobias, Miles, and Wesley. Observers recorded data using paper and pencil for Garrett and Graham. The dependent variable was the duration, measured in seconds, of mask compliance (a timer was used when data were collected via paper and pencil). Mask compliance was defined as the mask covering the participant's mouth and nose simultaneously with the elastic bands positioned behind each ear or, for masks that tied, knotted bows that rested at the back of the neck and crown of the head. Brief moving of the mask was permitted (e.g., for the participant to wipe or scratch their nose), provided that they did not remove the mask (defined in what follows) and they pulled the mask back over their mouth and nose within $5 \mathrm{~s}$ of being prompted to "wear the mask." Data on the amount of time the participant wore the mask before it was removed were collected as a duration measure.

Data were also collected on the frequency of successful mask removals, blocked attempts to remove the mask (during treatment phases only), and targeted problem behavior. Mask removal was defined as the participant pulling the mask below the mouth and/or nose and not moving it back up within $5 \mathrm{~s}$ of being prompted to do so, or pulling the mask away from the face such that the elastic that was previously positioned behind the ears, or knots positioned on the neck and head, came loose, exposing the nose and mouth. Blocked attempts were recorded during treatment for Eleanor, Tobias, Miles, and Wesley. Due to a miscommunication, blocking data were not recorded for Garrett. Blocking data were not recorded for Graham, as he did not experience the treatment phase. Blocked attempts were defined as the participant bringing their hand to the mask and the therapist physically intervening by placing their hand between the participant's hand and the mask, then gently guiding the participant's hand away from the mask. Targeted problem behaviors, which were defined individually for each participant, generally included aggression, disruptive behavior, inappropriate vocalizations, and/or self-injurious behavior. Aggression included attempting to or successfully hitting, punching, pulling hair, kicking, scratching, pinching, forcefully grabbing the limbs or clothing of others, or throwing objects within $0.61 \mathrm{~m}$ of a person. Disruptive behavior generally included attempting to or successfully breaking, ripping, swiping, and/or throwing objects (not within $0.61 \mathrm{~m}$ of a person); causing identifiable damage to the environment; and attempting to cause damage that would be successful in a home setting (e.g., banging on a window). Inappropriate verbalizations included cursing and threatening harm to others. Self-injurious behavior included attempting to or successfully hitting any part of the body or head/face with an open or closed fist, poking eyes, and forcefully scratching or pinching any part of the body or head/face.

\section{Interobserver Agreement}

A second observer simultaneously, but independently, collected data during a mean of $28.7 \%$ of sessions (range $19.4 \%$ $40 \%$ ) for Miles, Tobias, Wesley, and Garrett. Interobserver data were not collected for Eleanor and Graham due to staffing restrictions resulting from the COVID-19 pandemic. Interobserver agreement for Miles, Tobias, and Wesley was calculated using the partial-agreement within-intervals method (Mudford et al., 2009). Specifically, after dividing each session into consecutive 10-s bins, the smaller frequency (or duration) of recorded responses in each bin was divided by the larger frequency (or duration); the quotient was then multiplied by 100 . Bins in which both data collectors indicated that zero responses occurred were given a value of 1 . Agreements were averaged across all 10-s bins. Mean agreement across participants equaled $95.7 \%$ (range $91.9 \%-98.5 \%$ ) for the duration of wearing the mask, $98.1 \%$ (range $94.2 \%-100 \%$ ) for the frequency of mask removals, $96.1 \%$ (range $89.7 \%-100 \%$ ) for blocked attempts to remove the mask, and $99.5 \%$ for the frequency of problem behavior (range $98.4 \%-100 \%$ ). Total session agreement was calculated for Garrett (i.e., the smaller duration or frequency was divided by the larger duration or frequency recorded by each data collector; the quotient was then multiplied by 100); agreement coefficients equaled $100 \%$ for each measure.

\section{Procedures}

Every session began with the therapist placing a mask on the participant's face, positioning it such that it covered the mouth and nose, and vocally instructing them to wear the mask. Participants were provided with an activity to engage in throughout all sessions. This included preferred toys for Eleanor, Tobias, and Wesley; attention for Miles; and various leisure and academic activities for Garrett and Graham. The preferred toys, talking (attention), and leisure items were identified via preference assessments conducted during the course of their admissions. The academic activities were those that were used during regular educational programming that participants engaged in during their inpatient admission. Sessions varied in duration and were terminated after the participant independently removed the mask.

\section{Baseline}

The therapist placed a cloth mask on the participant's face, and the participant was instructed to keep the mask on. They 
were presented with activities in which to engage. No other contingencies were in place (e.g., they did not receive reinforcement for wearing the mask, and mask removal was not blocked). As noted previously, Graham was compliant with wearing the mask during baseline and wore it for extended durations without the need for additional intervention. Therefore, he did not participate in the treatment phase described next.

\section{Treatment}

During treatment, attempts to remove the mask prior to the criterion duration elapsing were blocked by the therapist placing their hand between the participant's hand and face and moving the participant's hand away from their face; attempts to remove the mask after the criterion was met were not blocked. During the session, the therapist redirected the participant to the available activity following blocking by placing the participant's hands on the activity while verbally directing them to engage with it. If blocking was unsuccessful and the participant removed the mask prior to the criterion being reached, the session was terminated. Additional preferred activities (identified through preference assessments earlier in their admissions) were added at the start of treatment for Miles (music played on a tablet) and Tobias (puzzles). Music was added for Miles to provide a physical stimulus to redirect him to following blocking (as attention was only available prior to this phase), and puzzles were added for Tobias to provide him with more activities to engage in as his engagement with the items available in baseline was sometimes variable. If the criterion elapsed without a successful mask removal, the therapist provided praise (e.g., "Great job wearing your mask!") and indicated reinforcement was available (by presenting the token board or showing the participant the food). Assistance with removing the mask was provided if the participant motioned to remove the mask or attempted to remove it but had difficulty taking it off. For Eleanor and Miles, a piece of preferred food (chocolate chip and Swedish fish, respectively) was delivered in addition to praise. If they removed the mask prior to the criterion duration elapsing, the session was terminated, the activity was removed, and the preferred food was withheld. Food was not included in the initial treatment evaluation for Tobias and Wesley as their problem behavior was previously demonstrated to be maintained by access to preferred foods. However, as described next, it was later added to promote compliance with mask wearing.

\section{Additional Treatment Procedures}

Several additional procedures were conducted to identify stimuli that competed with mask removal (Eleanor) or to teach token earning for wearing the mask (Tobias and Wesley). Details of these procedures are described in what follows.
Eleanor A competing stimulus assessment (CSA; Piazza et al., 1998) was conducted to identify stimuli that would compete with mask removal. Sessions were $5 \mathrm{~min}$ in duration, and each session began with the therapist placing the mask on Eleanor. Attempts to remove the mask were not blocked, and the session continued until the 5 min elapsed regardless of whether she was wearing the mask. During the control condition, Eleanor and the therapist walked throughout the hallways of the inpatient unit; no consequences were provided for wearing (or not wearing) the mask. Walking was selected as the activity as that best replicated the conditions Eleanor would experience in the community while having to wear her mask. Test sessions were identical to baseline; however, Eleanor also had access to a single item throughout the session. Six different items were assessed, one at a time, including a stuffed unicorn, a stuffed fox, bubble wrap, a rain stick, a play phone, and weighted gloves worn on Eleanor's hands. Data were collected on the duration of mask wearing and engagement with the test stimuli (or wearing the gloves). Stimuli that were associated with the highest levels of engagement and the longest durations of mask wearing were then provided noncontingently in the mask-tolerance treatment during a probe of the 15-min terminal session duration.

Tobias and Wesley A differential reinforcement of other behavior (DRO) treatment was incorporated into Tobias's and Wesley's treatment evaluations. During the DRO, tokens were delivered for not removing the mask for prespecified intervals that were systematically increased over time. The tokens included a picture of a cookie cut into thirds for Tobias and laminated smiley faces and a picture of candy as the terminal token for Wesley. Following token training, the DRO interval for mask removal was initially $10 \mathrm{~s}$ for Tobias and $20 \mathrm{~s}$ for Wesley; the participants earned one token for every $10 \mathrm{~s}$ or $20 \mathrm{~s}$ in which they did not remove their masks. After they earned three tokens, Tobias and Wesley were permitted to exchange the tokens for a small piece of preferred food (Oreo cookies for Tobias and Swedish fish for Wesley). Thus, Tobias and Wesley were initially required to wear the mask for a total of $30 \mathrm{~s}$ and $60 \mathrm{~s}$ (to earn all three tokens), respectively, before the opportunity to exchange the tokens became available. The DRO interval was then doubled for Tobias, or increased by $50 \%$ for Wesley, as they were successful in wearing the mask, until they reached the terminal criterion. For Tobias, this equaled a DRO of $10 \mathrm{~min}$; at the 10-min DRO, Tobias was required to wear the mask for 30 consecutive min to earn all three tokens, which he then exchanged for his food. For Wesley, the terminal DRO interval was scheduled to equal $3 \mathrm{~min}$ and $33 \mathrm{~s}$ such that he would have been required to wear the mask for 10 consecutive min to earn all three tokens. 


\section{Component Analysis}

After the terminal duration for mask compliance was reached, a component analysis was conducted with Miles, Tobias, and Garrett to determine whether certain treatment components were necessary to maintain the terminal mask compliance goal (component analyses were not conducted with the remaining participants due to time constraints). Miles's treatment included continuous attention, continuous music, and delivery of a preferred food after $30 \mathrm{~min}$ of wearing his mask. During the component analysis, continuous attention was removed first. Then, continuous music was removed, leaving only the delivery of the preferred food after 30 min of mask compliance. For Tobias, treatment included continuous access to preferred activities and the 10-min DRO for token delivery, with food being delivered after he earned three tokens. During the component analysis, the DRO was removed to determine whether access to activities alone would support consistent mask compliance. For Garrett, the treatment included completion of regularly scheduled activities (e.g., academics, leisure) and contingent edible reinforcement. During the component analysis, the edible reinforcement was removed.

\section{Results}

Graham complied with wearing the mask while performing various activities in his daily schedules. Therefore, additional intervention was not required. Supplemental Figure 1 depicts baseline data for Graham.

Eleanor, Miles, Tobias, Wesley, and Garrett were less compliant with consistently wearing the mask during everyday activities. Therefore, a CCD was used to gradually increase tolerance to longer durations of mask wearing and evaluate the use of contingent reinforcement for meeting the criterion. Figure 1 depicts the results for Eleanor, Miles, and Garrett. During baseline, Eleanor wore the mask, on average, for 24.7 s. Therefore, the initial criterion for earning reinforcement in treatment was $25 \mathrm{~s}$. Eleanor met this criterion in the final three sessions of this phase. The duration for which she was required to wear the mask was then increased by $50 \%$; Eleanor met the criterion consistently until it was increased to $86 \mathrm{~s}$. Eleanor began attempting to remove and successfully removing the mask more frequently at longer durations; however, with sufficient exposure, she eventually met the criterion at $86 \mathrm{~s}$ and $129 \mathrm{~s}$. Attempts to touch or remove the mask were variable throughout the treatment evaluation; however, very high frequencies or blocked attempts were observed during the latter sessions at $129 \mathrm{~s}$ and during the initial sessions at 194 s. Therefore, at the 194-s criterion, Eleanor was permitted to walk during the session to replicate conditions in which she was likely to wear the mask in the community (e.g., at stores). Blocked attempts decreased when she was permitted to walk,
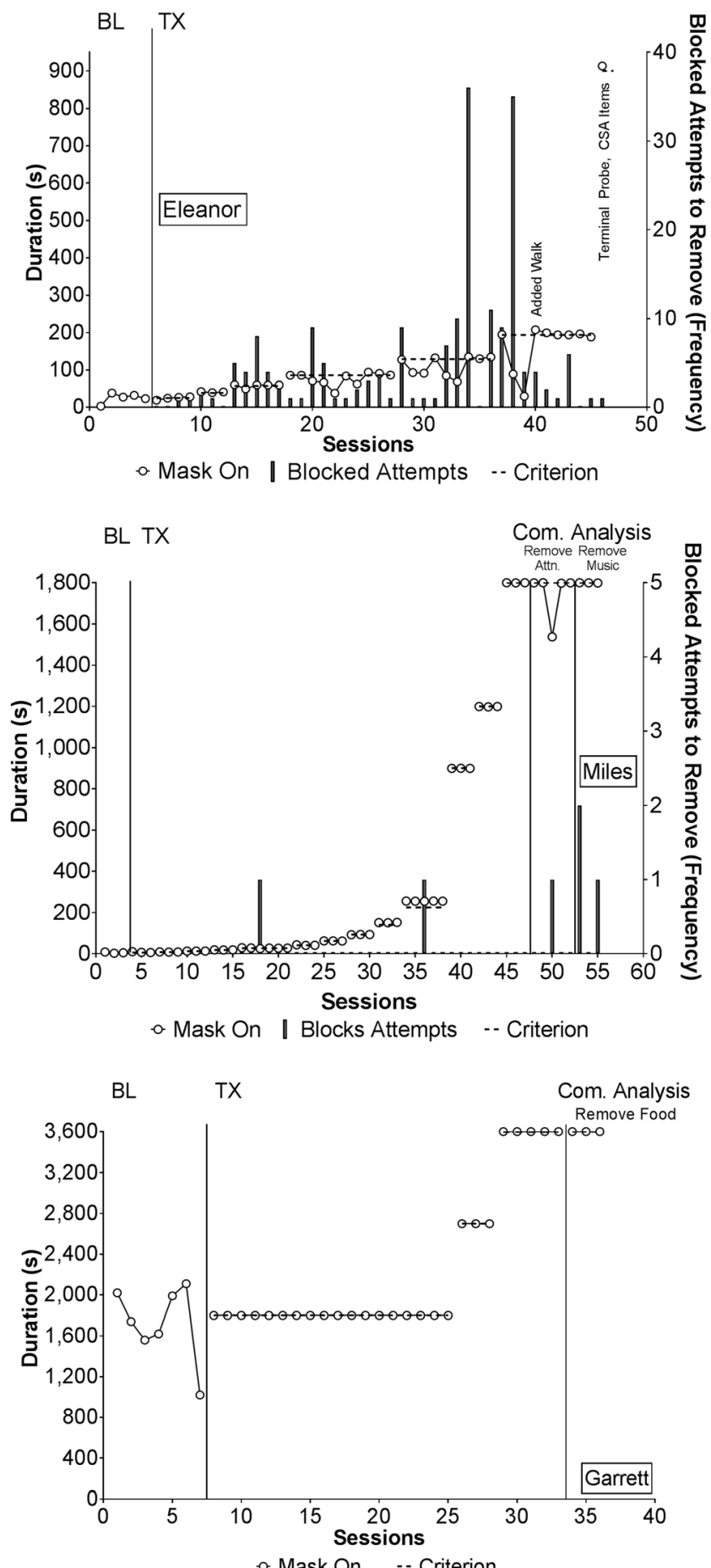

Fig. 1 Duration of Mask Wearing for Eleanor, Miles, and Garrett. Note. $\mathrm{BL}=$ baseline$; \mathrm{TX}=$ treatment $\mathrm{CSA}=$ competing stimulus assessment; Com. Analysis $=$ component analysis; Remove Attn. $=$ remove attention during component analysis

and remained relatively lower throughout the remainder of the evaluation. Following the completion of a CSA, a probe at the terminal session duration of 15 min was conducted. During this session, Eleanor had access to all of the items evaluated during the CSA (as they all successfully competed with 
attempts to remove the mask). She was permitted to choose which item(s) she wanted on her walk and could change items at any time. Eleanor wore her mask for the entire $15 \mathrm{~min}$, indicating that the delivery of preferred food and access to competing items were successful in maintaining her mask compliance. Eleanor did not engage in problem behavior during baseline. She engaged in a single instance of problem behavior in only two sessions during treatment, when the criterion was increased to $194 \mathrm{~s}$. Problem behavior was not correlated with blocked attempts to remove the mask.

Miles wore his mask during baseline for an average of 5.2 s. Therefore, the initial criterion was set at $5 \mathrm{~s}$. Miles successfully wore his mask as the criterion was increased by $50 \%$ until it reached $225 \mathrm{~s}$. At that point, larger increases in the duration criterion were made until he was wearing the mask for 1,800 $\mathrm{s}$ (30 $\mathrm{min}$ ). A component analysis was conducted to determine whether the continuous access to attention and music was necessary to maintain mask compliance. Miles continued to wear the mask despite the removal of both components. We decided not to remove the preferred food contingent on meeting the criterion to maintain some amount of reinforcement for mask compliance. Thus, delivery of preferred food alone was found to be sufficient to maintain mask compliance for Miles. Miles rarely attempted to remove his mask during treatment, doing so once during two separate treatment sessions, and one to two times across three sessions during the component analysis. Miles did not engage in problem behavior during baseline. He engaged in problem behavior in only two sessions during treatment; three instances of problem behavior were observed during a single session when the duration criterion equaled $27 \mathrm{~s}$, and one instance of problem behavior was observed during a single session when the criterion was $1,800 \mathrm{~s}$. In no case was problem behavior associated with blocking attempts to remove the mask.

During baseline, Garrett wore the mask for an average of $28.7 \mathrm{~min}(1,722 \mathrm{~s}$; range $17 \mathrm{~min}$ to $35 \mathrm{~min})$; therefore, the initial criterion was set at $30 \mathrm{~min}$. The duration of mask wearing immediately stabilized once the intervention was initiated. Given the variability in mask wearing observed during baseline, prolonged exposure to the 30 -min criterion was conducted to ensure he would consistently wear the mask for $30 \mathrm{~min}$ over multiple consecutive sessions. The criterion was then increased to $45 \mathrm{~min}$ and finally to the target of $60 \mathrm{~min}$, with Garrett successfully and consistently meeting each criterion within three sessions. Garrett continued to wear the mask when the edible reinforcement component was removed. Garrett did not engage in any problem behavior during baseline or treatment. Data were not collected on blocking; however, anecdotally, he never attempted to remove the mask during treatment.

Figure 2 depicted the results for Tobias and Wesley. During baseline, Tobias initially wore his mask for $15 \mathrm{~min}$; however, the duration of mask wearing became more variable
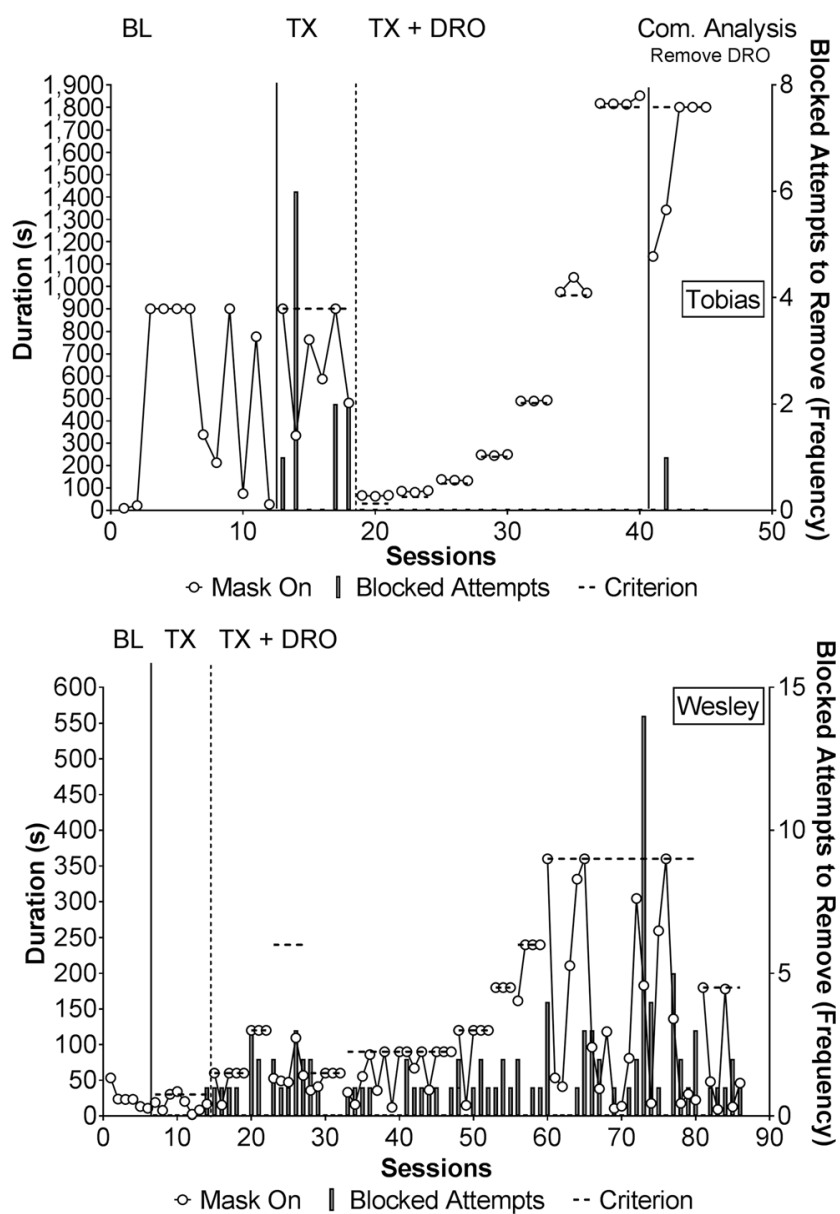

Fig. 2 Duration of Mask Wearing for Tobias and Wesley Note. $B L=$ baseline; $T X=$ treatment; $\mathrm{DRO}=$ differential reinforcement of other behavior; Com. Analysis = component analysis

over time. The initial criterion was then set for $15 \mathrm{~min}$ (as he had tolerated 15 min of wearing his mask over multiple sessions in baseline), and the efficacy of blocking and redirecting to the available activities was evaluated. The duration of mask wearing remained variable, and Tobias attempted to remove the mask at least once and up to four times across most sessions. Therefore, the DRO was added, and the criterion was reduced to $30 \mathrm{~s}$. Tobias successfully wore the mask for the duration of the $30 \mathrm{~s}$; therefore, the criterion was increased, doubling the amount of time he was required to wear the mask each time. This continued until he was wearing the mask for 30 min. At that point, the DRO was removed to determine whether mask wearing would maintain in its absence. Although the duration of wearing the mask initially decreased following the removal of the DRO, it quickly increased across sessions, and mask wearing maintained for up to $30 \mathrm{~min}$ with just access to various preferred activities. Tobias never attempted to remove the mask with the DRO in place, and only one blocked attempt to remove the mask occurred during the component analysis. Tobias did not engage in any problem behavior during baseline or treatment. 
Wesley's tolerance for wearing the mask decreased across sessions in baseline, despite having access to preferred toys. The initial criterion was set at $30 \mathrm{~s}$, during which time attempts to remove the mask were blocked. Wesley often removed the mask quickly such that the therapist did not have time to block and redirect him to the toys. Therefore, the DRO was added. Following the addition of the DRO, compliance with wearing the mask increased until the criterion was increased to $240 \mathrm{~s}$. Wesley frequently attempted to remove the mask and was ultimately successful in taking it off in each session at this duration such that he never met this criterion. Therefore, the duration was decreased back to $60 \mathrm{~s}$. Thereafter, the criterion was increased by $50 \%$, rather than doubled. Although attempts to remove the mask persisted, blocking was more successful, and Wesley met each criterion until it was increased to 360 s. At that point, attempts to remove the mask increased. The duration of mask wearing became quite variable, and he eventually began successfully removing the mask at durations that approximated his baseline performance. An attempt to reverse to a shorter duration, $180 \mathrm{~s}$, was made; however, mask wearing remained variable. Therefore, this evaluation was discontinued, and other methods to promote mask tolerance were pursued.

\section{Discussion}

Behavioral interventions have been proven highly effective in treating a wide variety of behavioral challenges for individuals with IDDs, including reducing severe problem behavior (Carr et al., 2009; Kurtz et al., 2011) and increasing tolerance to medical procedures (McComas et al., 1998; Riviere et al., 2011). The results of the current study indicate that behavioral intervention may also be effective in increasing compliance with wearing a mask for a particularly vulnerable population, a true imperative during an unusually turbulent time in our history. For Graham, simply being prompted to wear a mask while he was engaged in a variety of ongoing activities (e.g., academics) was sufficient to attain mask compliance. For the other five participants, results indicated arranging reinforcement for a slowly changing criterion was successful in increasing the duration of mask compliance. However, for one of these individuals (Wesley), the duration of mask wearing did not maintain. These results provide additional support for the use of behavioral interventions to increase compliance with health care routines for many individuals with IDDs, allowing them and their families to still access their community during these unprecedented times.

These results also suggest that the CCD may be a useful approach to slowly build tolerance for those who refuse to wear masks without the need for added treatment components; it also allows clinicians to determine at what criterion additional components may be required if an individual consistently fails to meet a given criterion (i.e., it promotes less restrictive measures in practice, as additional intervention can be implemented precisely at the point at which the individual demonstrates difficulty tolerating the specific criterion). These additional components, such as competing items or DRO, may be required initially to promote increased tolerance, but results also indicate they may be faded out or discontinued after the terminal goal is met (i.e., they are required to get over the "hump" the individual is stuck at during tolerance training but can be discontinued once tolerance is sufficiently established). A component analysis was conducted with several of the participants after they met their terminal goal, and the additional components used to help increase compliance were deemed nonessential and removed from the treatment. This is encouraging as it suggests that lesser response effort on the part of the caregivers is possible.

Attempts to remove the mask were variable across participants. Miles and, reportedly, Garrett rarely tried to remove the mask during treatment. For Eleanor, Tobias, and Wesley, who more frequently attempted to remove the mask, additional components were required. Walking and competing stimuli were effective at decreasing mask removal attempts for Eleanor, and the DRO was effective for Tobias. However, Wesley's mask removal attempts, and successes, persisted even with the inclusion of the DRO. Ultimately, the intervention was not effective in producing sustained mask wearing for Wesley. It is unclear why, although one possibility is that his frequent attempts to remove the mask were intermittently reinforced with escape when those attempts were not successfully blocked and the session was ultimately terminated. It is possible Wesley learned that repeated attempts to remove the mask would eventually be reinforced with escape from the session. This theory is bolstered by the fact that compliance with mask wearing was eventually obtained with Wesley in a subsequent treatment evaluation by employing escape extinction such that mask removal no longer resulted in the termination of the session. Rather, if Wesley took off his mask, the experimenter simply placed it back on his face and continued with the session. Eventually, after several sessions with exposure to this new contingency, the frequency of mask removal decreased, and Wesley eventually met the 10-min criterion for mask wearing. These data are not depicted in the current study but are available upon request.

This study is not without limitations. For one, although the overall design was the same and contingent edible reinforcement was used in each case for Eleanor, Miles, Tobias, Wesley, and Garrett, other components were added for some participants, such as tokens and competing stimuli. These differences may limit generalizability across participants; however, the outcomes for four of the five participants who underwent treatment support the use of the CCD to implement stepwise changes in criteria to build tolerance to potentially aversive stimuli. 
There were also some limitations associated with the use of the CCD. Although experimental control was demonstrated across multiple successive phases in Eleanor's CCD, only a single probe was conducted at the terminal duration when the stimuli from the CSA were added. The CSA was conducted because it was noted that, although Eleanor was wearing the mask for upward of $200 \mathrm{~s}$, removal attempts were increasing in frequency, and it was not feasible for caregivers to block removals at such a high rate while in the community. Although only a single session was conducted within the mask-tolerance intervention with the competing stimuli, it is important to note that the CSA itself is an experimental analysis in which the effects of each individual test stimulus on the dependent variable are compared to the control condition in which no stimulus is available (Hagopian et al., 2020). During the CSA for Eleanor (see Supplemental Figure 2), three consecutive series of the control and test sessions were conducted, with the session duration equaling $5 \mathrm{~min}$. Variable durations of mask wearing were observed during the control series $(M=$ $161.1 \mathrm{~s}$, range $45.9 \mathrm{~s}$ to $297 \mathrm{~s}$ ), compared to very high and stable durations of mask wearing, which were observed across sessions with five of the six test stimuli $(M=298.8 \mathrm{~s}$, range $297 \mathrm{~s}$ to $300 \mathrm{~s}$ ). Thus, replication of the effects of the test stimuli on mask wearing was achieved within the CSA. After the CSA was completed, Eleanor's pending discharge from the unit was rapidly approaching; therefore, the terminal goal was probed using the competing stimuli to further replicate the outcomes of the CSA and to evaluate the efficacy of the competing stimuli during an extended session duration.

In addition, meeting the criterion at each phase of the CCD was signaled by the experimenter providing praise and a piece of food or token and no longer blocking mask removals. Though the experimenter did not force the participant to remove his or her mask, these signals could have had the effect of artificially capping the session at the criterion duration as (a) the consumption of the food and proper mask wearing (covering the mouth and nose) were mutually exclusive and (b) participants typically responded by removing (or attempting to remove, sometimes requiring assistance) their masks following the presentation of the piece of food or terminal token exchangeable for the food. Thus, it is possible that the use of food in the context of this design may have resulted in greater adherence to the criteria than would have been achieved with some other reinforcer that did not similarly compete with proper mask wearing.

Graham did not complete the treatment portion of the study protocol; therefore, his data do not necessarily contribute to an understanding of the generality of the study procedures. Given that he was compliant with wearing the mask for durations that exceeded the terminal goal of $60 \mathrm{~min}$, the intervention was deemed unnecessary; however, we note this success for two reasons. First, we wanted to report on all cases in which the intervention was attempted in accordance with guidelines for a consecutive controlled case series (Hagopian, 2020).
Second, the inclusion of Graham further highlights that some individuals with IDDs, even those with long histories of engaging in severe problem behavior, can be compliant with wearing face masks in the absence of additional intervention. Therefore, a diagnosis of an IDD or the presence of severe problem behavior should not deter caregivers, practitioners, and educators from evaluating compliance with wearing a face mask.

Future research should focus on alleviating the limitations identified in this study. First, experimenters should determine which components are the most effective, necessary, and sufficient. The current study used noncontingent access to activities, contingent reinforcement, response blocking, competing stimuli (Eleanor), and a DRO with tokens (Tobias and Wesley). A component analysis was not conducted with Eleanor and Garrett due to time constraints. Although a component analysis was conducted with Miles and Tobias, food was not removed from Miles's intervention, and response blocking was not removed from either (although it should be noted that neither participant attempted to remove the mask frequently during the latter criteria). Second, if additional components are added to a CCD, a reversal should be conducted to strengthen experimental control.

Finally, future research should consider safety modifications and monitoring for mask compliance and usage in those individuals with IDDs and comorbid medical conditions that may complicate mask usage, such as asthma, lung disease, craniofacial abnormalities, or other respiratory pathology.

Overall, these six individuals were given an increased opportunity for safety, independence, and access to their community by learning to tolerate wearing a mask during the COVID-19 pandemic. Additionally, although four participants required additional components, the additions were noncomplex and effective (competing stimuli for Eleanor, music for Miles, and DRO for Tobias), and several were able to be removed for two participants (music for Miles and DRO for Tobias) after a component analysis. Together, these results offer a glimmer of light in a time of darkness and remind us that small victories, like tolerating a mask, can lead to big triumphs for those individuals with IDDs.

Supplementary Information The online version contains supplementary material available at https://doi.org/10.1007/s40617-021-00583-7.

\section{Declarations}

Conflict of interest The authors declare they have no conflict of interest.

\section{References}

Ather, B., Mirza, T. M., \& Edemekong, P. F. (2020). Airborne precautions. StatPearls Publishing. https://www.ncbi.nlm.nih.gov/ books/NBK531468/ 
Bullock, C. E., Fisher, W. W., \& Hagopian, L. P. (2017). Description and validation of a computerized behavioral data program: "BDataPro". The Behavior Analyst, 40(1), 275-285. https://doi.org/10.1007/ s40614-016-0079-0.

Carr, J. E., Severtson, J. M., \& Lepper, T. L. (2009). Noncontingent reinforcement is an empirically supported treatment for problem behavior exhibited by individuals with developmental disabilities. Research in Developmental Disabilities, 30(1), 44-57. https://doi. org/10.1016/j.ridd.2008.03.002.

Centers for Disease Control and Prevention. (2020). How to protect yourself \& others. https://www.cdc.gov/coronavirus/2019-ncov/preventgetting-sick/prevention.html

Cheng, V. C., Wong, S. C., Chuang, V. W., So, S. Y., Chen, J. H., Sridhar, S., To, K. K., Chan, J. F., Hung, I. F., Ho, P. L., \& Yuen, K. Y. (2020). The role of community-wide wearing of face mask for control of coronavirus disease 2019 (COVID-19) epidemic due to SARS-CoV-2. Journal of Infection, 81(1), 107-114. https://doi.org/ 10.1016/j.jinf.2020.04.024.

Chu, D. K., Akl, E. A., Duda, S., Solo, K., Yaacoub, S., \& Schünemann, H. J. (2020). Physical distancing, face masks, and eye protection to prevent person-to-person transmission of SARS-CoV-2 and COVID-19: A systematic review and meta-analysis. The Lancet, 395(10242), 1973-1987. https://doi.org/10.1016/S0140-6736(20) 31142-9.

Courtenay, K., \& Perera, B. (2020). COVID-19 and people with intellectual disability: Impacts of a pandemic. Irish Journal of Psychological Medicine, 37(3), 231-236. https://doi.org/10.1017/ ipm.2020.45.

DeLeon, I. G., Hagopian, L. P., Rodriguez-Catter, V., Bowman, L. G., Long, E. S., \& Boelter, E. W. (2008). Increasing wearing of prescription glasses in individuals with mental retardation. Journal of Applied Behavior Analysis, 41(1), 137-142. https://doi.org/10.1901/ jaba.2008.41-137.

Eikenberry, S. E., Mancuso, M., Iboi, E., Phan, T., Eikenberry, K., Kuang, Y., Kostelich, E., \& Gumel, A. B. (2020). To mask or not to mask: Modeling the potential for face mask use by the general public to curtail the COVID-19 pandemic. Infectious Disease Modelling, 5, 293-308. https://doi.org/10.1016/j.idm.2020.04.001.

Flood, W. A., \& Wilder, D. A. (2004). The use of differential reinforcement and fading to increase time away from a caregiver in a child with separation anxiety disorder. Education and Treatment of Children, 27(1), 1-8 https://www.jstor.org/stable/42899780.

Hagopian, L. P. (2020). The consecutive controlled case series: Design, data-analytics, and reporting methods supporting the study of generality. Journal of Applied Behavior Analysis, 53(2), 596-619. https://doi.org/10.1002/jaba.691.

Hagopian, L. P., Frank-Crawford, M. A., Javed, N., Fisher, A. B., Dillon, C. M., Zarcone, J. R., \& Rooker, G. W. (2020). Initial outcomes of an augmented competing stimulus assessment. Journal of Applied Behavior Analysis, 53(4), 2172-2185. https://doi.org/10.1002/jaba. 725 .

Johns Hopkins University \& Medicine. (n.d.). Coronavirus resource center. Retrieved September 24, 2020, from https://coronavirus. jhu.edu/

Kahng, S. W., Boscoe, J. H., \& Byrne, S. (2003). The use of an escape contingency and a token economy to increase food acceptance. Journal of Applied Behavior Analysis, 36(3), 349-353. https://doi. org/10.1901/jaba.2003.36-349.

Kurtz, P. F., Boelter, E. W., Jarmolowicz, D. P., Chin, M. D., \& Hagopian, L. P. (2011). An analysis of functional communication training as an empirically supported treatment for problem behavior displayed by individuals with intellectual disabilities. Research in Developmental Disabilities, 32(6), 2935-2942. https://doi.org/10. 1016/j.ridd.2011.05.009.

McComas, J. J., Wacker, D. P., \& Cooper, L. J. (1998). Increasing compliance with medical procedures: Application of the highprobability request procedure to a toddler. Journal of Applied Behavior Analysis, 31(2), 287-290. https://doi.org/10.1901/jaba. 1998.31-287.

McDougall, D., Hawkins, J., Brady, M., \& Jenkins, A. (2006). Recent innovations in the changing criterion design: Implications for research and practice in special education. Journal of Special Education, 40(1), 2-15. 10.1177\%2F00224669060400010101.

Mudford, O. C., Martin, N. T., Hui, J. K. Y., \& Taylor, S. A. (2009). Assessing observer accuracy in continuous recording of rate and duration: Three algorithms compared. Journal of Applied Behavior Analysis, 42(3), 527-539. https://doi.org/10.1901/jaba.2009.42527.

O’Leary, L., Hughes-McCormack, L., Dunn, K., \& Cooper, S. (2018). Early death and causes of death of people with Down syndrome: A systematic review. Journal of Applied Research in Intellectual Disabilities, 31(5), 687-708. https://doi.org/10.1111/jar.12446.

Piazza, C. C., Fisher, W. W., Hanley, G. P., LeBlanc, L. A., Worsdell, A. S., Lindauer, S. E., \& Keeney, K. M. (1998). Treatment of pica through multiple analyses of its reinforcing functions. Journal of Applied Behavior Analysis, 31(2), 165-189. https://doi.org/10. 1901/jaba.1998.31-165.

Puts, N. A., Wodka, E. L., Tommerdahl, M., Mostofsky, S. H., \& Edden, R. A. (2014). Impaired tactile processing in children with autism spectrum disorder. Journal of Neurophysiology, 111(9), 18031811. https://doi.org/10.1152/jn.00890.2013.

Riviere, V., Becquet, M., Peltret, E., Facon, B., \& Darcheville, J. C. (2011). Increasing compliance with medical examination requests directed to children with autism: Effects of a high-probability request procedure. Journal of Applied Behavior Analysis, 44(1), 193-197. https://doi.org/10.1901/jaba.2011.44-193.

Shapiro, J. (2020). COVID-19 infections and deaths are higher among those with intellectual disabilities [Special series report]. In Morning Edition. NPR. https://n.pr/2QNhLU6

Stuesser, H. A., \& Roscoe, E. M. (2020). An evaluation of differential reinforcement with stimulus fading as an intervention for medical compliance. Journal of Applied Behavior Analysis, 53(3), 16061621. https://doi.org/10.1002/jaba.685.

Sunjaya, A. P., \& Jenkins, C. (2020). Rationale for universal face masks in public against COVID-19. Respirology, 25(7), 678-679. https:// doi.org/10.1111/resp.13834.

Turk, M. A., Landes, S. D., Formica, M. K., \& Goss, K. D. (2020). Intellectual and developmental disability and COVID-19 case-fatality trends: TriNetX analysis. Disability and Health Journal, 13(3), 100942. https://doi.org/10.1016/j.dhjo.2020.100942.

Wheatley, T. L., Goulet, M., Mann, K., \& Lanovaz, M. J. (2019). Differential negative reinforcement of other behavior to increase compliance with wearing an anti-strip suit. Journal of Applied Behavior Analysis, 53(2), 1153-1161. https://doi.org/10.1002/jaba. 632.

Publisher's Note Springer Nature remains neutral with regard to jurisdictional claims in published maps and institutional affiliations. 